\title{
Dislocation of the penis: a rare complication after traumatic pelvic injury
}

\author{
Mei Chin $\underline{\operatorname{Lim}}^{1}$, MBBS, FRCR, Sivasubramanian $\underline{\text { Srinivasan }}^{1}$, MD, FRCR, Hui Seong $\underline{T e h}^{1}$, MBBS, FRCR,
} Chang Peng Colin ${\underline{T e O^{2}}}^{2}$ MBBS, FEBU

\begin{abstract}
Traumatic injury to the male external genitalia is frequently encountered, but acute traumatic dislocation of the penile structure is extremely rare, with only a few reports found in the literature. We herein report the case of a 21-year-old man who sustained blunt trauma to the pelvis following a motor vehicle accident, and had features suspicious of penile dislocation. With the use of computed tomography and bedside ultrasonography, a diagnosis of penile dislocation was made, which was subsequently confirmed intraoperatively. Immediate surgical intervention via gentle manipulation of the penile tissue back to its native position was performed in order to restore normal anatomy. The exact mechanism of penile dislocation is not known. However, circumferential laceration around the foreskin causing degloving injury of the penis is suggested in our patient.
\end{abstract}

Keywords: degloving injury of penis, dislocation of penis, male genital trauma

\section{INTRODUCTION}

Dislocation of the penis is an unusual condition, with only a few cases reported in the literature, all occurring after trauma. ${ }^{(1,2)}$ This condition arises as a result of abnormal force translated to the penile structure. Patients often experience acute penile pain after trauma. Diagnosis can be suspected, in the setting of appropriate history of acute groin injury, when the penile tissue cannot be palpated in the expected position, with an abnormal enlargement of the scrotal sac. Diagnosis is confirmed when the penis is displaced and visualised in an ectopic location, such as within the scrotum. Gentle manipulation of the displaced penis back to its native position under general anaesthesia is the mainstay treatment.

\section{CASE REPORT}

Our patient was a 21-year-old male motorcyclist who presented to the emergency department following a head-on collision with a car. He was flung forward and his groin struck the gas tank in front of the motorcycle. At presentation, he complained of severe groin and perineal pain. He had a significant past medical history of congenital cryptorchidism of the left testis, but did not seek any former urological consultation with regard to his condition. Physical examination revealed severe tenderness in the scrotum and left inguinal region. The cutaneous sheath of the penis was visualised externally in entirety, but the bulk of the penile tissue could not be palpated. There was abnormal fullness in the right scrotal sac. The left testis was not palpated in the left scrotal sac. No significant bleeding was detected. Secondary survey according to the advanced trauma life support protocol revealed no other injury except for superficial abrasions in the forehead and lower limbs.

Laboratory investigations were unremarkable. Computed tomography (CT) of the head, thorax, abdomen and pelvis was performed as per trauma protocol, which showed that the bulk of the penile tissue was not in the expected position; rather, it appeared to be displaced into the right scrotal sac (Fig. 1). The right testis was in the normal position, with grossly preserved morphology. There was a heterogeneously enhancing soft tissue mass in the left inguinal region, likely to represent the maldescended testis. The morphology of the left testis appeared deformed and was suspicious for testicular rupture. A mildly displaced right superior pubic ramus fracture was also seen.

In view of the CT findings, bedside ultrasonography was performed to exclude penile fracture and to assess the viability of the penis. The penis was visualised in the right hemiscrotum (Fig. 2) and appeared intact, with normal vascularity. There was no evidence of penile fracture. The right testis was normal in size, morphology and vascularity. However, the left testis could not be visualised in the left hemiscrotum. Ultrasonography of the left inguinal region was not performed due to patient discomfort.

Emergency surgery was performed, which confirmed that the penile shaft was displaced into the right scrotum. No fracture of the corpus cavernosum is detected. The penis was gently manipulated and relocated to its original position without difficulty (Fig. 3). Circumcision was performed for incidental phimosis. The right testis was intact in the right scrotum. The left testis was seen in the left inguinal canal. Exploration of the left testis was performed via a median raphe incision and revealed severe contusion of the left testis with no viable bleeding. As there was more than $50 \%$ rupture of the testis with extrusion of the seminiferous tubules, a decision for left orchidectomy was made.

The convalescent phase was uneventful and the patient was discharged on the third postoperative day.

\section{DISCUSSION}

The penis comprises three erectile columns, namely the corpus spongiosum and paired corpora cavernosa. It is surrounded 

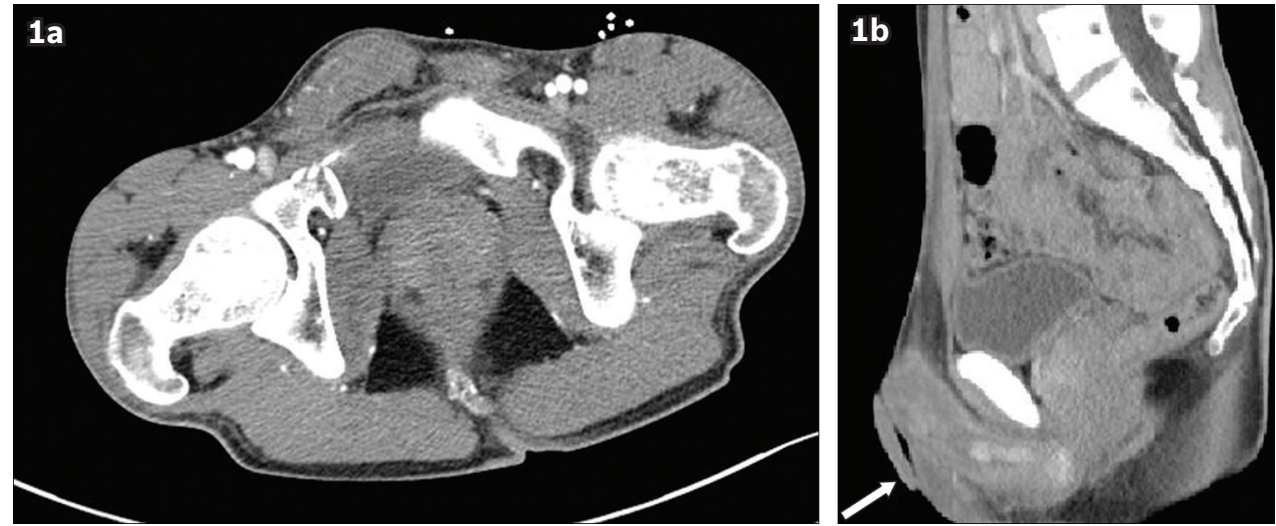

Fig. 1 CT images of the pelvis show penile tissue displaced in the right scrotum. (a) In the axial view, a mildly displaced comminuted fracture of the right superior pubic ramus is seen, and (b) in the sagittal view, only loose skin (arrow) is visualised in the expected position of the penis.

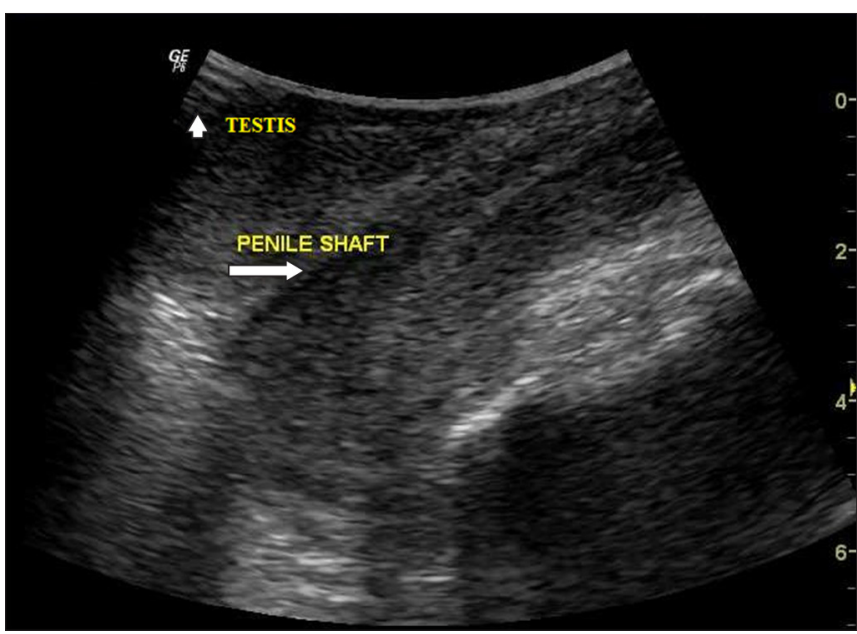

Fig. 2 Ultrasonography image (transducer probe placed over the righ scrotum) shows the displaced penile shaft (arrow) within the scrotal sac. The penile shaft tissue appears contiguous, with no evidence of fracture, and the right testis (arrowhead) is displaced laterally to the penis.

by fascial layers, nerves, lymphatics and blood vessels, and covered by skin. ${ }^{(3)}$ The penis is supported at its base by the penile suspensory ligament.

Penile dislocation is an exceedingly rare entity, occurring as a result of either blunt or penetrating pelvic trauma. Little is known about this condition, with only a few cases reported in the literature. Acute perineal pain is the most encountered complaint. Other clinical findings include penile hypermobility and instability. Associated complications, which include erectile dysfunction and urethral injury, are uncommon.

The mechanism of penile dislocation is poorly understood. In our patient, we hypothesise that due to the severe impact on the pelvis and an abnormally translated force to the penis, there was a violent traction of the penile tissue backwards, causing abrupt circumferential coronal laceration around the prepuce. This resulted in the cutaneous sheath of the penis being separated from the underlying penile tissue, with the degloved penile tissue displaced to an ectopic location. The penis may be displaced into the scrotum, groin or in front of the pubis. ${ }^{(1)}$ Our patient had underlying phimosis, which contributed to the increased propensity for preputial tear following high-impact trauma. The high-impact trauma also caused a fracture of the right superior
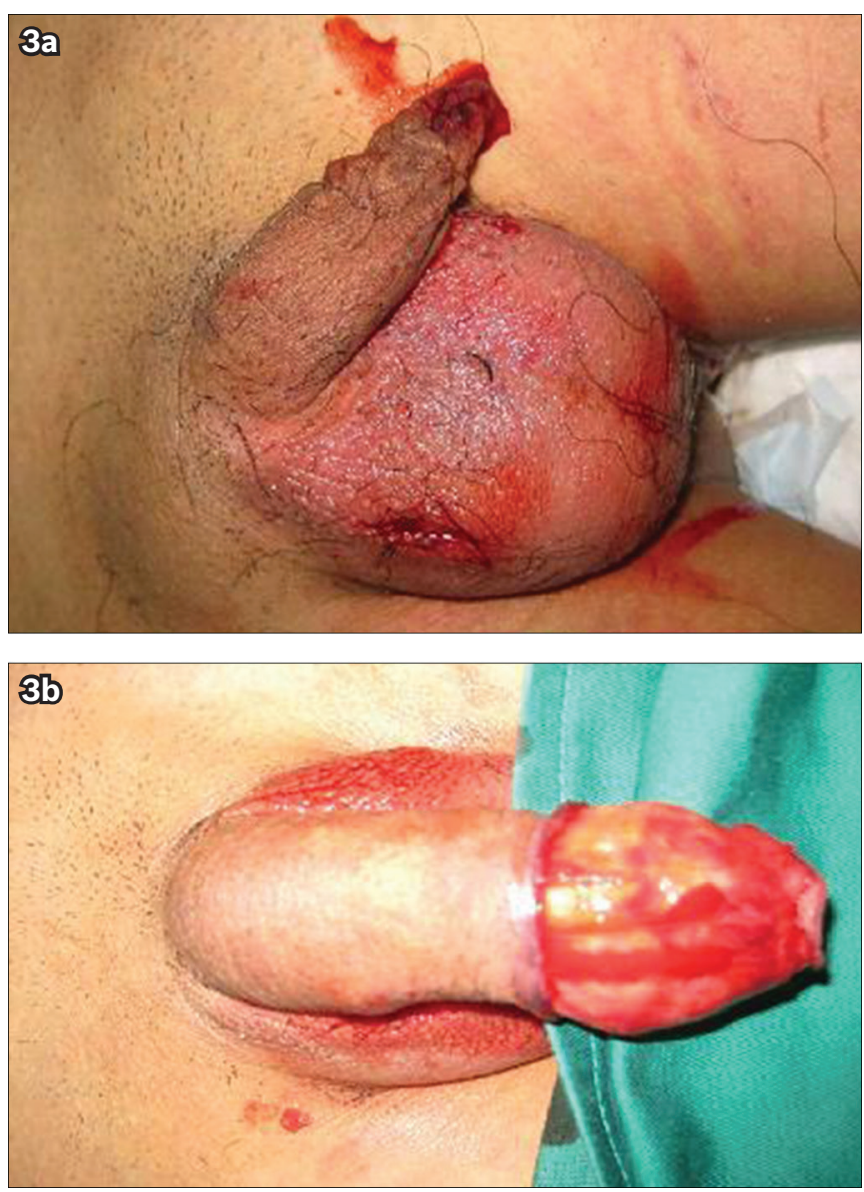

Fig. 3 Photographs of the dislocated penis show (a) only the loose skin of the penis and abnormally enlarged right scrotal sac; and (b) the result of gentle manipulation of the penile tissue back into its cutaneous sheath.

pubic ramus. We hypothesise that the left inguinal testis might have absorbed most of the impact and prevented a left pubic ramus fracture; consequently, the impact also resulted in rupture of the left pubic ramus and subsequent orchidectomy.

Although no previous papers have discussed the use of imaging modalities in the evaluation of penile dislocation, we opine that ultrasonography will be useful, particularly in the emergency setting, to confirm the presence of penile tissue in ectopic location. Ultrasonography, in conjunction with the use of duplex Doppler, is also useful in confirming the viability of 
penile structures and assessing the scrotal contents. ${ }^{(4)}$ However, ultrasonography has its accompanying limitation of being heavily operator dependent. CT is usually performed as part of trauma protocol and can be used for the visualisation of the ectopic penis, as well as detect any associated pelvic injury. Magnetic resonance (MR) imaging has little value in the emergency setting; however, it may be performed in selected patients to excluded penile fracture.

Penile dislocation can be readily managed with surgical intervention. Careful manipulation of the displaced penile tissue under general anaesthesia is an effective method of treatment. Penile dislocation should be distinguished from the relatively more common penile fractures. Penile fracture refers to the rupture of the corpus cavernosum, typically in an erect penis. Most cases of penile fracture occur after vigorous sexual intercourse, and less frequently, associated with trauma. Patients may experience a characteristic 'clicking' sound during coitus with immediate detumescence as well as swelling, haematoma formation, penile deformity and deviation of the penis to the contralateral side. The fracture is typically seen as a transverse corporal tear on the ventral portion of the penis close to the urethra. ${ }^{(5)}$ Penile tissue is not displaced from its anatomical location in penile fractures, and associated urethral injury is common. ${ }^{(6)}$

Penile fracture is primarily a clinical diagnosis, particularly with a convincing history. Imaging modalities such as corpus cavernosography, ultrasonography and MR imaging may be considered in patients with equivocal findings. Cavernosography characteristically demonstrates extravasation from the corpus cavernosum following intracorporal contrast injection. ${ }^{(7)}$
Ultrasonography is a good noninvasive alternative with no ionising radiation. Dynamic evaluation of the integrity of the penile shaft can be performed. However, small tears in the tunica albuginea may be easily overlooked, creating false negative results. ${ }^{(8)} \mathrm{MR}$ imaging is also another noninvasive alternative to detect corporal disruption. On T1-weighted sequence, hyperintense vascular sinusoids can be distinguished from hypointense fibrous tunica albuginea. Any disruption of the tunica albuginea is suspicious for penile fracture. Surrounding haematoma can also be easily detected. Although MR imaging is more sensitive than cavernosography and ultrasonography, it is time consuming and technically difficult to perform in an emergency setting. ${ }^{(8)}$ No case of concomitant penile fracture and dislocation has been reported so far.

\section{REFERENCES}

1. Curr JF. Dislocation of the penis. Br J Urol 1946; 18:66.

2. Shiraki IW, Trichel BE. Traumatic dislocation of the penis. J Urol 1969; 101:186-8.

3. Jordan $\mathrm{GH}$, Schlossberg SM. Surgery of the penis and urethra. In: Walsh PC, Retik AB, Vaughan ED Jr, Wein AJ, eds. Campbell's Urology, 8th ed. Philadelphia: Saunders 2002: 3886-98.

4. Avery LL, Scheinfeld MH. Imaging of penile and scrotal emergencies. Radiographics 2013; 33:721-40.

5. Chang AJ, Brandes SB. Advances in diagnosis and management of genital injuries. Urol Clin North Am 2013; 40:427-38.

6. Pandyan GV, Zaharani AB, Al Rashid M. Fracture penis: An analysis of 26 cases. ScientificWorldJournal 2006; 6:2327-33.

7. Pliskow RJ, Ohme RK. Corpus caverosonography in active fracture of the penis. AJR Am J Roentgenol 1979; 133:331-2.

8. Morey AF, Metro MJ, Carney KJ, Miller KS, McAninch JW. Consensus on genitourinary trauma: external genitalia. BJU Int 2004; 94:507-515. 